\title{
From 'perfect mix' to 'potion magique' - regulatory T cells and anti-inflammatory cytokines as adjuvant targets: reply from Guy
}

\section{Bruno Guy}

The proposal of Bayry and colleagues to replace adjuvant approaches, such as Toll-like receptor (TLR) stimulation, with the inhibition of regulatory $\mathrm{T}\left(\mathrm{T}_{\mathrm{Reg}}\right)$ cells, or to combine both strategies, is an attractive one. I would nevertheless suggest that this strategy would be best reserved for therapeutic vaccines, in situations where the negative role of $\mathrm{T}_{\mathrm{Reg}}$ cells on protective immunity is well established (for example, in some chronic diseases such as parasitic diseases or tuberculosis ${ }^{1,2}$ ).

As vaccine developers, we can try to mimic pathogens in their initiation of immune responses, but we must be cautious of interfering too much with natural regulatory mechanisms, and in particular the feedback mechanisms that prevent over-reactions. For example, $\mathrm{T}_{\mathrm{Reg}}$ cells are essential for preventing autoimmunity; the role of some TLR agonists in inducing these disorders has been discussed $^{3}$, and any potential risk of this occuring should be limited.

Bayry and colleagues also propose the use of interleukin-10 (IL-10) suppression, but I feel this should also be reserved for therapeutic settings. Again, nature itself usually does things properly, and inflammatory T helper 1 cells have recently been shown to secrete IL-10 as a feedback mechanism ${ }^{4}$. Preventing this feedback could lead to over-reactive inflammatory responses. Although the goal, target and mechanism were different, the TGN1412 'disaster' showed us that interfering directly with regulatory mechanisms can be dangerous.

In conclusion, the approaches proposed by Bayry and colleagues could be powerful but the outcomes are difficult to predict precisely. For this reason, I would be cautious about using them in the routine vaccination of healthy infants (where even rare side-effects are not acceptable) and would reserve them, at least initially, for use in therapeutic vaccination against cancer ${ }^{5}$, or chronic infections, for which the benefits might outweigh the risks.

Research Department, sanofi pasteur, Campus Merieux, Marcy l'Etoile 69280, France. e-mail:bruno.guy@sanofipasteur.com

doi: 10.1038/nrmicro 1681-c2

1. Belkaid, Y., Sun, C. M. \& Bouladoux, N. et al. Parasites and immunoregulatory T cells. Curr. Opin. Immunol. 18, 406-412 (2006).

2. Scott-Browne, J. P. et al. Expansion and function of Foxp3-expressing $T$ regulatory cells during tuberculosis. J. Exp. Med. 204, 2159-2169 (2007)

3. Marshak-Rothstein, A. Toll-like receptors in systemic autoimmune disease. Nature Rev. Immunol. 6 , 823-835 (2006).

4. Trinchieri, G. Interleukin-10 production by effector T cells: Th1 cells show self control. J. Exp. Med. 204, 239-243 (2007).

5. Curiel, T. J. Tregs and rethinking cancer immunotherapy. J. Clin. Invest. 117, 1167-1174 (2007). 